$$
\text { CONf- } 960859--4
$$

\title{
Thermodynamic and Kinetic Consistency of Calculated Binary Nucleation Rates
}

G. Wilemski

B. E. Wyslouzil
RECENDE JUN 271996

OSTI

This paper was prepared for submittal to the

14th International Conference on

Nucleation and Atmospheric Aerosols

Helsinki, Finland

August 26-30, 1996

April 2, 1996

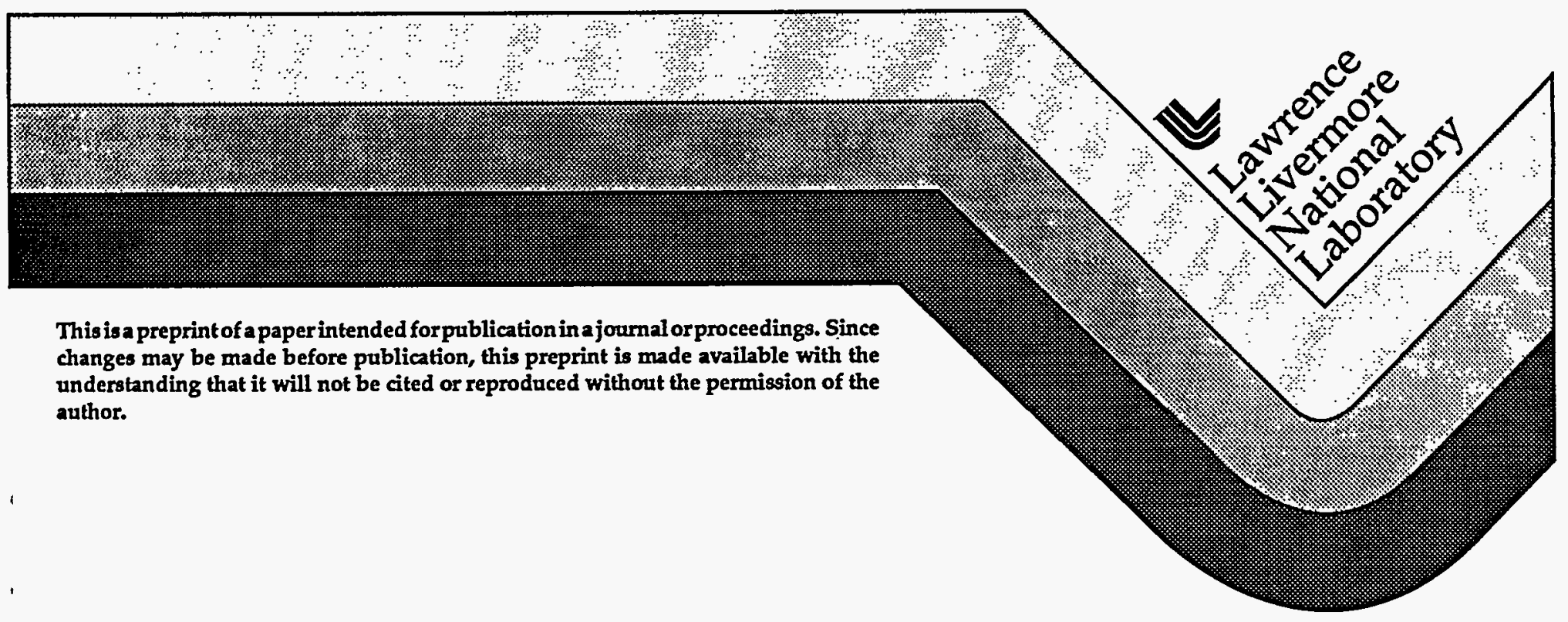

This is a preprint of a paper intended for publication in a journal or proceedings. Since changes may be made before publication, this preprint is made available with the understanding that it will not be cited or reproduced without the permission of the author.

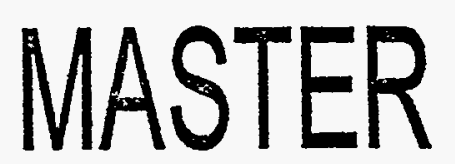

OISTRIBUTON OF THS DOCUAEAT IS LELIMTED DCC 


\section{DISCLAIMER}

This document was prepared as an account of work sponsored by an agency of the United States Government. Neither the United States Government nor the University of California nor any of their employees, makes any warranty, express or implied, or assumes any legal liability or responsibility for the accuracy, completeness, or usefulness of any information, apparatus, product, or process disclosed, or represents that its use would not infringe privately owned rights. Reference herein to any specific commercial product, process, or service by trade name, trademark, manufacturer, or otherwise, does not necessarily constitute or imply its endorsement, recommendation, or favoring by the United States Government or the University of California. The views and opinions of authors expressed herein do not necessarily state or reflect those of the United States Government or the University of California, and shall not be 1 sed for advertising or product endorsement purposes. 


\title{
THERMODYNAMIC AND KINETIC CONSISTENCY OF CALCULATED BINARY NUCLEATION RATES
}

\author{
GERALD WILEMSKI \\ Lawrence Livermore National Laboratory, Livermore, CA 94551-9900 USA \\ and \\ BARBARA E. WYSLOUZIL \\ Worcester Polytechnic Institute, Worcester, MA 01609-2280 USA
}

\begin{abstract}
To establish the accuracy and applicability of analytical expressions for the steady state rate of binary nucleation, we numerically solved the birth-death equations for the vapor-to-liquid transition. These calculations were performed using rate coefficients that are consistent with the principle of detailed balance and a new self-consistent form of the equilibrium distribution function for binary cluster concentrations. We found that the customary saddle point and growth path approximations are almost always valid and can fail only if the nucleating solution phase is significantly nonideal. For example, problems can arise when the vapor composition puts the system on the verge of partial liquid phase miscibility. When this occurs for comparable monomer impingement rates, nucleation still occurs through the saddle point, but the usual quadratic expansion for the cluster free energy is inadequate. When the two impingement rates differ significantly, however, the major particle flux may bypass the saddle point and cross a low ridge on the free energy surface. The dependence of the saddle point location on the gas phase composition is also important in initiating or terminating ridge crossing nucleation.
\end{abstract}

\section{INTRODUCTION}

The availability of accurately measured binary nucleation rates now makes it possible to critically test approximate theoretical rate expressions. Before performing these assessments, it is important to understand how the predicted rates are affected by their inherent assumptions and approximations so that any lack of agreement with experiment can be properly attributed to either physical failure of the theory or poor-mathematical approximations. The key ideas to test concern whether the main nucleation current flows through the saddle point in the direction of steepest descent (Reiss, 1950), in the growth direction (Stauffer, 1976), or avoids the saddle point. The reliability of the customary parabolic expansion for the cluster free energy should also be checked. At present these tests can be made only by comparing the approximate rate predictions with exact numerical solutions of the birth-death equations for binary nucleation kinetics. Such comparisons should be made as consistently as possible with respect to the primary ingredients used in each approach, namely, the equilibrium distribution of binary cluster concentrations and the forward and reverse rate constants governing the rates at which clusters add and lose monomers. These quantities are related by the equations of detailed balance, but these relationships alone do not prevent inconsistencies from arising (Wilemski and Wyslouzil, 1995). At a minimum, the following requirements should be met: The equilibrium distribution should obey the law of mass action, and it should also display appropriate limiting behavior as one component vanishes. The evaporation (reverse) rate coefficients should be unique functions of cluster composition, and they should not depend unphysically on the monomer vapor concentrations or total pressure. Forward rate constants are determined straightforwardly from the kinetic theory of gases and are not problematic. The best way to ensure consistency is through a microscopic approach such as density functional theory (Zeng and Oxtoby, 1991). Unfortunately, these theories are currently impossible to apply to most substances of interest, and the 
only recourse is.to more phenomenological approaches, usually based on the classical capillarity approximation (CA). Despite its deficiencies, the CA still provides the most practical means to make routine rate predictions. The results presented here are based on a new version of the binary equilibrium cluster size distribution (Wilemski and Wyslouzil, 1995) that still uses the $\mathrm{CA}$ but satisfies all of the consistency and limiting requirements just noted. Other CA-based distributions (Reiss, 1950; Kulmala et al., 1992) do not.

Previous numerical studies of the binary birth-death equations have treated only ideal mixtures (Greer et al., 1990; Kožíšek and Demo, 1993, 1995) or the nonideal mixtures $\mathrm{H}_{2} \mathrm{SO}_{4}-$ $\mathrm{H}_{2} \mathrm{O}$ and $\mathrm{NH}_{3}-\mathrm{H}_{2} \mathrm{O}$ (Vehkamäki et al., 1994; McGraw, 1995). Successes and failures of the saddle point approximation were reported, and cases of saddle point avoidance were found by Greer et al. (1990) and McGraw (1995), but only limited conclusions about the validity of different analytical rate theories can be drawn from these studies. This paper highlights some results from our recent numerical study of binary nucleation in six systems representing many types of ideal and nonideal solution behavior and covering a wide range of conditions (Wyslouzil and Wilemski, 1995). Our focus here is on numerical and analytical rate comparisons for two model positively deviating systems PD1 and PD2. The physical properties of PD1 and PD2 are those of, respectively, the ideal o-xylene-m-xylene and ethanol-hexanol mixtures with an excess free energy of mixing that is quadratic in the mole fractions $x, g^{E}=A x_{A} x_{B}$. Ethanol and hexanol form ideal liquid mixtures even though the equilibrium vapor pressures of the pure components differ by a factor of 226 at our simulation temperature of $260 \mathrm{~K}$. We put $A=2 R T$ at each simulation temperature to force each system to be at its upper critical solution temperature (UCST), just on the verge of phase separation into two solution phases.

\section{RESULTS AND DISCUSSION}

For the PD1 system, analytical nucleation rates calculated using the expressions of Reiss and Stauffer generally agreed well with each other and with the numerically calculated rates over many orders of magnitude of variation in the impingement rate ratio $r$. It is generally expected that the Reiss and Stauffer expressions should disagree markedly at very large or small values of $r$. Because the equilibrium vapor pressures of the pure components here are very similar, these extreme conditions drive the saddle point very close to a pure component axis leaving no "room" for difference between the Reiss and Stauffer flow directions. Although the flow of the main nucleation current through the saddle point is thoroughly confirmed for this system, a failure of the parabolic approximation for the free energy of cluster formation is illustrated in Fig. 1. As the vapor activity of species $B$ is varied, the saddle point composition passes very near, if not through, the value at the microscopic UCST. This causes the free energy surface to flatten and broaden and considerably reduces both the true curvature of the free energy surface and the effective curvature in Stauffer's theory. (The first three compositional derivatives of the molar free energy of mixing are zero at the bulk UCST critical composition.) As a result, the similar rate predictions, $J_{S}$ and $J_{R}$, of the Stauffer and Reiss theories become unreasonably large in this region. The figure also shows that a simple modification of Stauffer's rate, denoted by $J_{W S}$, remains fairly well-behaved and in reasonable agreement with the numerical results, since it is better at handling this kind of singular behavior as explained by Wyslouzil and Wilemski (1995).

In Fig. 2, the numerical and analytical rates are compared for the PD2 system. There are extensive ranges of vapor activity over which the numerical rates agree much better with Stauffer's theory than with Reiss', but there are also regions where the numerical rates disagree with both analytical theories. In contrast to the PD1 system, the predictions of Stauffer and Reiss can now differ because for this system the great difference in the equilibrium vapor pres- 


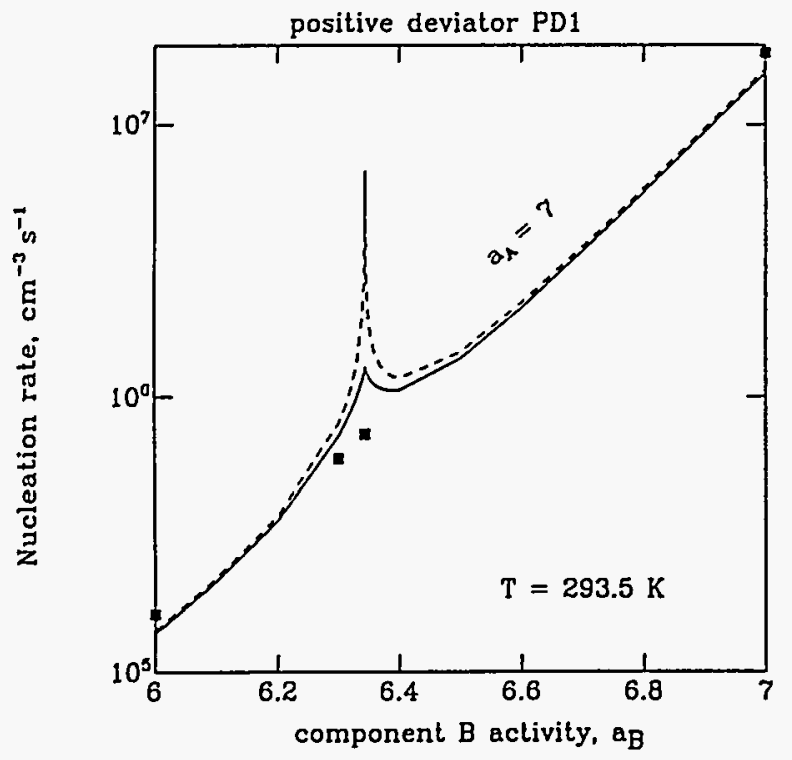

Figure 1. Steady state nucleation rates for the PD1 system based on o-xylene(A)$\mathrm{m}$-xylene(B) near a microscopic UCST. Numerical results (filled squares) are compared to the analytical predictions of $J_{W S}$ (solid line) and $J_{S}$ (dashed line).

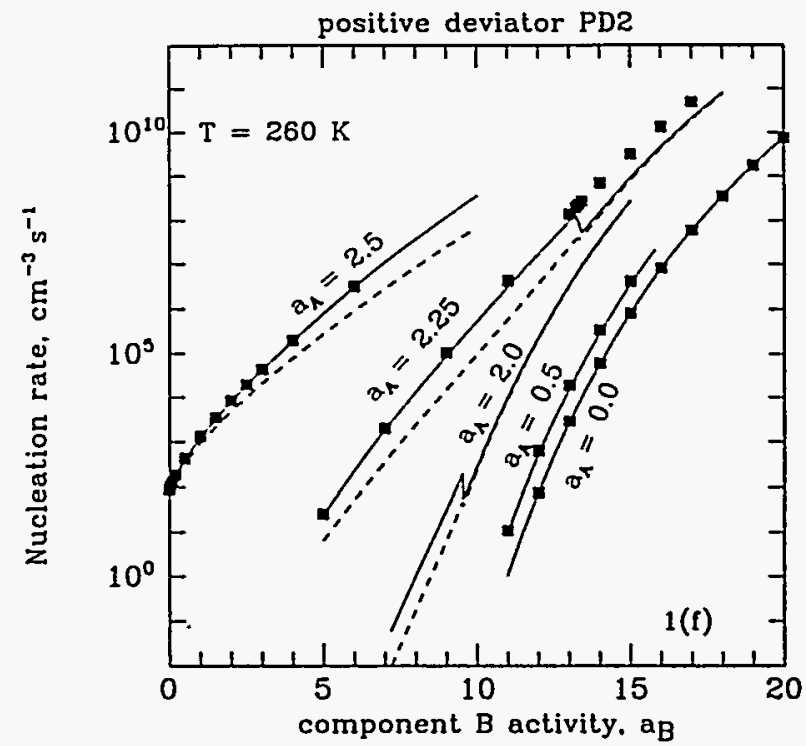

Figure 2. Numerical nucleation rates (filled squares) and analytical predictions of $J_{W S}$ (solid lines) and $J_{W R}$ (dashed lines) for the PD2 system based on ethanol(A)-hexanol(B). The results for $a_{A}=2.0$ indicate another region $\left(a_{B} \geq 9.6\right)$ where ridge crossing is expected.

sures makes it possible to have large values of $r$ while the component activities remain roughly comparable. This places the saddle point at a reasonable distance from either pure component axis and permits differences in the growth and steepest descent direction to arise as illustrated in Fig. 3(a). In Fig. 2, we also see that increasing the A-to-B impingement rate ratio by reducing $a_{B}$ at constant $a_{A}(=2.5)$, leads to a convergence of the two rate predictions because the saddle point moves ever closer to the $A$ axis leaving essentially only one direction through the saddle. Thus, the size of $r$ alone is insufficient to generate a significant difference between the Reiss and Stauffer theories. The location of the saddle point on the free energy surface plays an important role as well, and this is strongly affected by the relative sizes of the equilibrium vapor pressures and other properties of the mixture.

At activities in Fig. 2 for which the analytical theories fail, the major flux avoids the saddle point and climbs over a low ridge, as in Fig. 3(b). Ridge crossing has theoretically been ascribed to coupling between the shape of the free energy surface and a large disparity in the impingement rates $(r>>1$ or $r<<1)$ (Trinkaus, 1983). Our result is evidence that the "dynamic" behavior of the free energy surface is also important for producing a situation favoring ridge crossing nucleation as $r$ is varied. The picture of a free energy surface that is fairly insensitive to changes in $r$ may be appropriate for condensed phase nucleation (Greer at al., 1990), but it is not realistic for gas phase nucleation. For gas-liquid systems, the saddle point location can be very sensitive to changes in the vapor composition and, hence, in $r$.

\section{ACKNOWLEDGMENTS}

This work was supported by the National Science Foundation under Grant No. CHE9502604 (BEW) and the U.S. Department of Energy, Office of Basic Energy Sciences, Division of Geosciences and Engineering (GW). Part of this work was performed under the auspices of the U.S. Department of Energy by the Lawrence Livermore National Laboratory under Contract No. W-7405-ENG-48. 

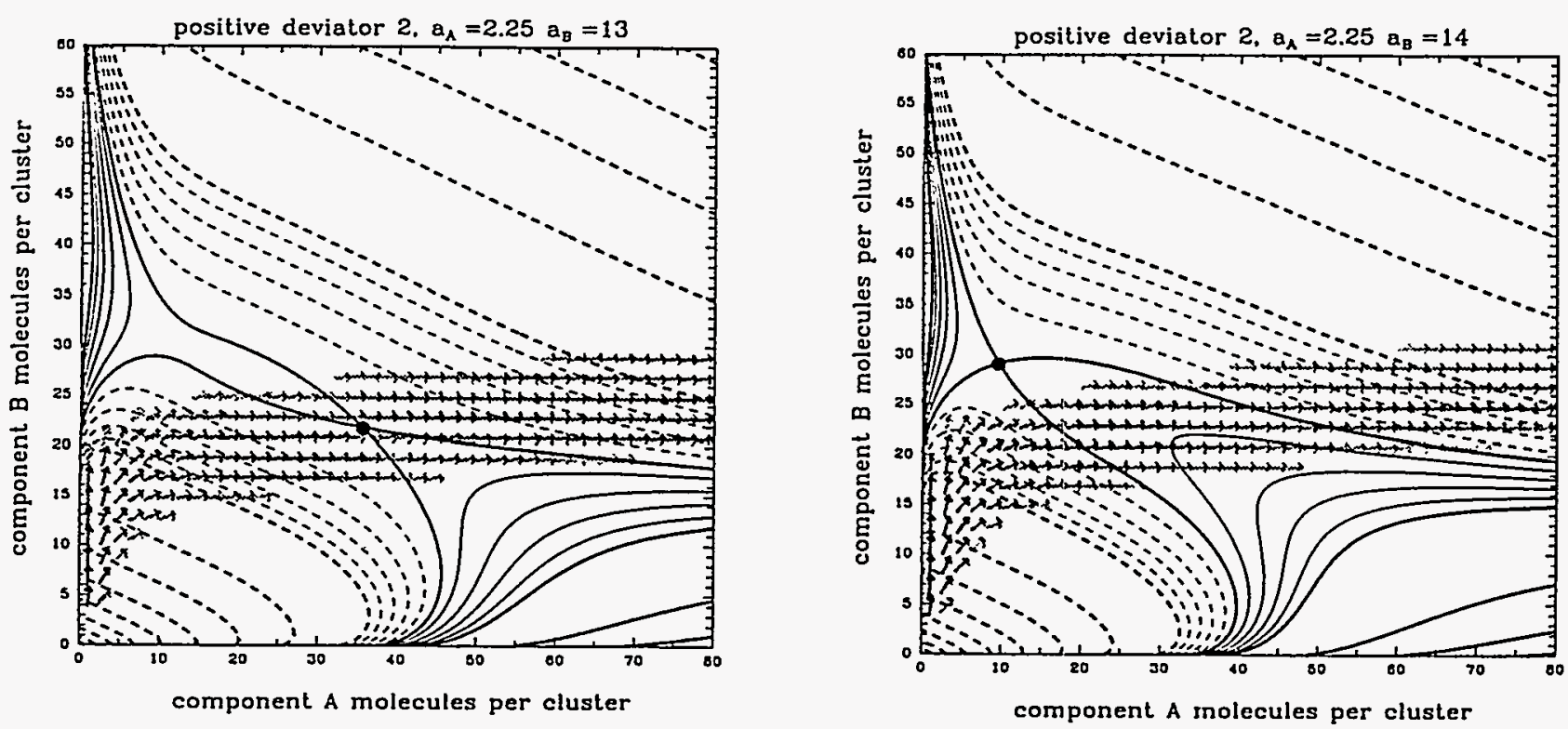

Figure 3. Steady state nucleation fluxes superimposed on a. contour plot of the free energy surface for the PD2 system at the indicated vapor activities. The saddle point is at the $\bullet$. The light contours are spaced at $0.5 k T$ intervals relative to the saddle point. The heavy contours start at $\pm 2.5 k T$ relative to the saddle and are then spaced at $5 k T$ intervals.

(a-left) $W /(k T)=47.7$ at the analytical saddle point. The major flux flows through the saddle region in a direction distinctly different from the path of steepest descent.

(b-right) $W /(k T)=45.5$ at the analytical saddle point. The major flux bypasses the saddle and climbs over a low ridge that extends from the saddle point toward the lower right corner.

\section{REFERENCES}

Greer, A.L., Evans, P.V., Hamerton, R.G., Shangguan, D.K., and Kelton, K.F. (1990) Numerical modeling of crystal nucleation in glasses, J. Cryst. Growth 99,38 .

Kožíšek, Z. and Demo, P. (1993) Transient kinetics of binary nucleation, J. Cryst. Growth 132, 491.

Kožíšek, Z. and Demo, P. (1995) Transient nucleation in binary ideal solution, J. Chem. Phys. 102,7595 .

Kulmala, M., Laaksonen, A., and Girshick, S.L. (1992) The self-consistency correction to homogeneous nucleation: Extension to binary systems, J. Aerosol Sci. 23, 309.

McGraw, R. (1995) Two-dimensional kinetics of binary nucleation in sulfuric acid-water mixtures, J. Chem. Phys. 102, 2098.

Reiss, H. (1950) The kinetics of phase transitions in binary systems, J. Chem. Phys. 18, 840. Stauffer, D. (1976) Kinetic theory of two-component ("heteromolecular") nucleation and condensation, J. Aerosol Sci. 7, 319 .

Trinkaus, H. (1983) Theory of the nucleation of multicomponent precipitates, Phys. Rev. B 27, 7372.

Vehkamäki, H., Paatero, P., Kulmala, M., and Laaksonen, A. (1994) Binary nucleation kinetics: A matrix method, J. Chem. Phys. 101, 9997.

Wilemski, G. and Wyslouzil, B.E. (1995) Binary nucleation kinetics. I. Self-consistent size distribution, J. Chem. Phys. 103, 1137.

Wyslouzil, B.E. and Wilemski, G. (1995) Binary nucleation kinetics. II. Numerical solution of the birth-death equations, J. Chem. Phys. 103, 1137.

Zeng, X.C. and Oxtoby, D.W. (1991) Binary homogeneous nucleation theory for the gas-liquid transition: A nonclassical approach, J. Chem. Phys. 95, 5940. 